\title{
Is it possible to detect in situ the sulfur loading of a fixed bed catalysts with a sensor?
}

\author{
P. Fremerey ${ }^{1,2}$, A. Jess ${ }^{2}$, and R. Moos ${ }^{1}$ \\ ${ }^{1}$ Department of Functional Materials, University of Bayreuth, Bayreuth, Germany \\ ${ }^{2}$ Department of Chemical Engineering, University of Bayreuth, Bayreuth, Germany \\ Correspondence to: R. Moos (funktionsmaterialien@uni-bayreuth.de)
}

Received: 30 October 2014 - Revised: 13 February 2015 - Accepted: 15 March 2015 - Published: 13 April 2015

\begin{abstract}
This study reports on a sensor concept to measure in situ sulfur poisoning (sulfidation) of refinery catalysts, in this case, of commercial silica pellets loaded with highly dispersed nickel. Catalyst pellets were poisoned in diluted $\mathrm{H}_{2} \mathrm{~S}$ between 100 and $400{ }^{\circ} \mathrm{C}$ and the sulfidation of the catalyst was observed. During this process, nickel sulfides are formed on the catalyst according to X-ray diffraction spectra and energy dispersive X-ray spectroscopy data. The sulfidation kinetics was quantitatively described by a shrinking core model. Representative catalyst pellets were electrically contacted, and their impedance was recorded in situ during sulfidation. At the beginning, the particles are highly insulating and behave capacitively. Their conductivity increases by decades during sulfidation. At high temperatures, an almost constant slope in the double-logarithmic representation vs. time can be found. At low temperatures, the conductivity remains constantly low for a long time but changes then rapidly by decades, which is also indicated by the phase that drops from capacitive to ohmic behavior. Since nickel sulfides exhibit a lower conductivity than nickel, the conductivity increase by decades during sulfidation can only be explained by electrically conducting percolation paths that form during sulfidation. They originate from the increased volume of sulfides compared to the pure nickel metal.
\end{abstract}

\section{Introduction}

In industry, heterogeneous catalysts are used to accelerate chemical reactions and to enhance product selectivity. During reaction, the catalysts are exposed to a harsh environment that may deactivate the catalyst. Sulfur compounds in the feed stream, such as hydrogen sulfide $\left(\mathrm{H}_{2} \mathrm{~S}\right)$, are a typical example. Thereby, $\mathrm{H}_{2} \mathrm{~S}$ chemisorbs either on the surface of the catalyst or it forms sulfides with the metal components. In both cases, this frequently leads to a deactivation of the active centers of the catalyst. For example, sulfur poisoning is typically observed during reforming of heavy gasoline, if the feedstock (low-octane gasoline) has not been deeply desulfurized previously (Oudar, 1980; Bartholomew et al., 1979). Another example is the nickel-catalyzed-steam reforming of hydrocarbons to syngas (Jess and Depner, 1999).

Today's standards in industry are ex situ methods to monitor the sulfur content of a fixed bed or even models that estimate it. They do not allow for an immediate response. Therefore, in situ methods are preferred to monitor the state of the catalyst directly. However, typical indirect methods based on spectroscopy are expensive and they do reflect the catalyst state only indirectly.

Therefore, a sensor that may detect the sulfur loading of a catalyst in situ was investigated in the present study. A nickel catalyst was poisoned with $\mathrm{H}_{2} \mathrm{~S}$ and the sulfidation of the catalyst was determined firstly locally resolved by EDX (energy dispersive X-ray spectroscopy). A chemical reaction model was applied to describe the sulfidation process quantitatively. After that, with a single-particle sensor, i.e., with a contacted representative catalyst particle, the sulfidation was measured in situ. In the subsequent section, it was investigated in detail how sulfur affects the particle conductivity. Finally, conductivity data, sulfidation results, and EDX data are compared. 


\section{Fundamentals and experimental methods}

\subsection{Experimental setup and parameters}

Figure 1 shows the laboratory setup that was used for all measurements. A thermally isolated quartz glass reactor was heated electrically. Inside of this reactor, a single-particle sensor was fixed in a glass holder at the lower end of the tube, where also the gas inlet was integrated. With this setup, several individual sensors were measured at constant experimental parameters. A gas flow of $50 \mathrm{Lh}^{-1}$ (NTP) was applied to the specimens. A bypass was installed in order to measure and adjust the inlet gas composition. Nitrogen was always used as a carrier gas. For the subsequent sulfidation, up to $1000 \mathrm{ppm}_{2} \mathrm{~S}$ were admixed to $\mathrm{N}_{2}$. Sulfidation was carried out from 100 to $350{ }^{\circ} \mathrm{C}$. For the reduction of the catalyst, $10 \% \mathrm{H}_{2}$ in $\mathrm{N}_{2}$ flowed through the system. The commercial catalyst (NiSAT 200, Clariant) consisted of porous cylindrical silica particles (length, $l=6 \mathrm{~mm}$; diameter, $d=6 \mathrm{~mm}$ ) loaded with approximately $37 \mathrm{wt} \%$ nickel (Kernchen et al., 2007). Since the sensor catalyst particle and the particles in the fixed bed were nominally identical, i.e., they were from the same batch, the changing electrical properties of the sensor particle reflect the behavior of the particles in the fixed bed. In other words, we can consider the sensor particle as a representative for the catalyst particles.

For each sulfidation run, a fresh sensor particle was used. Prior to sulfidation, the particles were brought into the reduced state by a preconditioning step, which was identical for each run. The sensor particle was heated up to $400^{\circ} \mathrm{C}$ under $\mathrm{N}_{2}$ so that no further nickel is converted to $\mathrm{NiO}$ until the measured electrical signal (see below) remained constant. After heating under $\mathrm{N}_{2}$, the catalyst was reduced with $10 \%$ hydrogen in $\mathrm{N}_{2}$ for $1 \mathrm{~h}$ to activate the entire catalyst material by reducing the existing $\mathrm{NiO}$ to $\mathrm{Ni}$. Then, the reactor was cooled to the desired temperature and the experiment started. Such conditioning procedures are carried out also in industrial applications. Therefore, it was applied for all particles to bring them into a defined state.

\subsection{Single-particle sensor and basic measurements}

For the sensor, the porous cylindrical catalyst particles were contacted electrically at the flat end planes. Two additional alumina pads that were coated on one side with gold were contacted with $0.1 \mathrm{~mm}$ gold wires. These two pads were pressed on the flat planes of the sensor particle; compare with Fig. 2. The other side of the alumina pads insulated the sensor particle against the steel casing, in which the sensor particle was fixed. The two gold wires were fed through the reactor by a four-bore alumina tube that was also fixed at the steel casing. The gold wires connected the sensor particle with the impedance analyzer (HP 4284A Precision LCR meter). Finally, the bores of the alumina tube were locked gastight with ceramic glue. A similar setup has been applied to de- tect coke formation on refinery catalysts in situ (Müller et al., 2010a, b).

From the recorded complex impedance data at $1 \mathrm{kHz}(\mathrm{ab}-$ solute impedance value $Z$, phase $\phi$ ), the resistances, $R$, were obtained by Eq. (1) (Macdonald, 1987), which is valid for a $\mathrm{R} \| \mathrm{C}$ behavior:

$R=Z / \cos (\varphi)$.

First, tests were conducted to exclude that any of the utilized materials and sensor components had a significant influence (negligible parallel resistance) on the signal during sulfidation. The following components were considered: the ceramic glue, the alumina tube, and the sensor without and with a particle. All results are summarized in Table 1. Experiments with the pure catalyst were not possible, since the catalyst could be purchased only in a nickel-loaded modification. However, in-house-prepared pressed pellets consisting of silicon oxide powder show a resistance which is in the range of the sensor with a particle at $450{ }^{\circ} \mathrm{C}$. Despite that the sensor resistance was 2 decades lower than the empty sensor holder, we could not exclude a priori that under some circumstances the parallel resistance may become non-negligible. Therefore, the empty sample holder was measured first at all temperatures and, by default, the impedances were corrected assuming a parallel impedance of sample and empty sample holder as described in Schönauer and Moos (2010).

From the resistance and the geometrical data, the conductivity, $\sigma$, was calculated.

$\sigma=\frac{1}{R} \cdot \frac{l}{A}$

Here $A$ is the area of the flat plane $\left(A=\pi d^{2} / 4 \approx 28.3 \mathrm{~mm}^{2}\right)$ and $l$ the length of the particle $(l=6 \mathrm{~mm})$. Occasional tests with a high-impedance analyzer (Novocontrol, type AlphaA) confirmed the results. Time-dependent impedance data were taken at $1 \mathrm{kHz}$ since this was the lowest possible frequency at which the current was high enough to ensure that crosstalk from the rough electrical environment did not predominate the signal. This was of special importance if the catalyst particle was in the reduced (high-ohmic) state.

Typical impedance plots in the complex plane in Fig. 3a (in the reduced, high-ohmic state) and in Fig. 3b (sulfidized) show that an $R \| C$ equivalent circuit is justified and that Eq. (1) can be applied.

\section{Results}

\subsection{Sulfidation of catalyst particle}

The sulfidation was at first investigated in detail ex situ at single particles by EDX analyses. The sulfidized sensor particles were ground from the flat plane to the center of the particle. Since the flat planes were covered by the gold layer, 


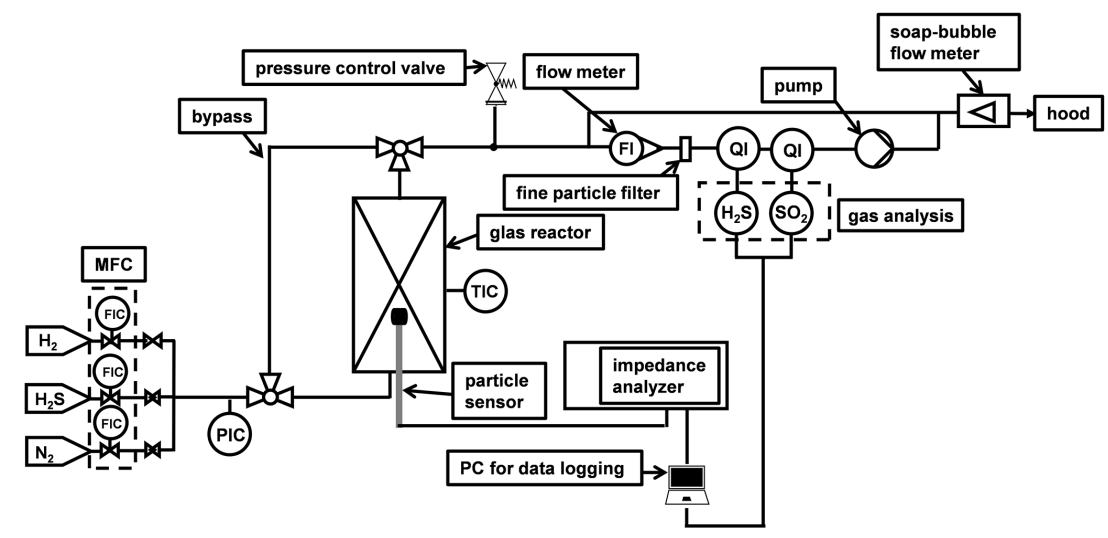

Figure 1. Scheme of the experimental setup.

Table 1. Electrical data of the sensor setup with and without a sensor particle. The resistance of the sensor particle is at least 2 decades lower than the sensor setup.

\begin{tabular}{lrrrl}
\hline Component & Temperature & Impedance in $\Omega$ & Phase in $^{\circ}$ & Resistance in $\Omega$ \\
\hline cables only & $\mathrm{RT}$ & $1.1 \cdot 10^{8}$ & -89.99 & $6.1 \cdot 10^{11}$ \\
+ ceramic glue & $\mathrm{RT}$ & $4.0 \cdot 10^{7}$ & -89.90 & $2.3 \cdot 10^{10}$ \\
+ ceramic tube & $450^{\circ} \mathrm{C}$ & $3.3 \cdot 10^{7}$ & -89.90 & $1.9 \cdot 10^{10}$ \\
+ sensor without particle & $450^{\circ} \mathrm{C}$ & $3.0 \cdot 10^{7}$ & -89.90 & $1.7 \cdot 10^{10}$ \\
+ sensor with particle & $450^{\circ} \mathrm{C}$ & $2.4 \cdot 10^{7}$ & -82.00 & $1.7 \cdot 10^{8}$ \\
\hline
\end{tabular}

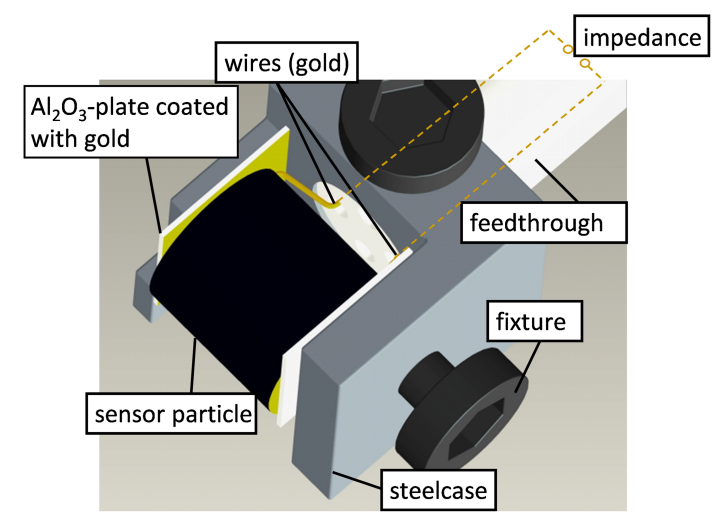

Figure 2. CAD drawing of the single-particle sensor.

the sulfidation occurred from the outer diameter of the cylindrical particles to the inner one. EDX line scans were conducted as shown in Fig. 4a. Line scans of samples sulfidized under $1000 \mathrm{ppm} \mathrm{H}_{2} \mathrm{~S}$ at $100{ }^{\circ} \mathrm{C}$ for 6 and $60 \mathrm{~h}$ are shown in Fig. $3 \mathrm{~b}$ and c, respectively. Results for samples treated at $300^{\circ} \mathrm{C}$ under $500 \mathrm{ppm} \mathrm{H}_{2} \mathrm{~S}$ for 2,24 , and $60 \mathrm{~h}$ are displayed in Fig. 5a-c. The proportions of nickel and sulfur are plotted in at. \%. A percentage of $50 \%$ nickel and $50 \%$ sulfur is a strong indication for the occurrence of the NiS phase. In contrast, a distribution of $60 \%$ nickel and $40 \%$ sulfur indicates $\mathrm{Ni}_{3} \mathrm{~S}_{2}$, a not fully sulfidized NiS phase. The zero points of the line scans are on the edge of the particles. As can be obtained from Fig. 4, the sulfur content is almost uniformly distributed from the outer diameter to the center of the catalyst particle. After $6 \mathrm{~h}$, a slow sulfur decrease from the outside to the inner side can be seen. After $60 \mathrm{~h}$, the sulfur is homogeneously distributed in the entire particle. This $\mathrm{Ni}: \mathrm{S}$ stoichiometry does not reach the $1: 1$ stoichiometry for NiS, but almost for $\mathrm{Ni}_{3} \mathrm{~S}_{2}$. Overall, the sulfidation proceeds almost homogeneously on the particle cross section at this low temperature. This suggests that the chemical reaction is the limiting factor in the sulfidation process at $100^{\circ} \mathrm{C}$ and not the (pore) diffusion of $\mathrm{H}_{2} \mathrm{~S}$ into the particle.

EDX data from the samples sulfidized at $300{ }^{\circ} \mathrm{C}$ (Fig. 5) look different. After 2, 24, and $60 \mathrm{~h}$, an almost vertical front moves into the particle. The $\mathrm{Ni}: \mathrm{S}$ ratio of nearly $1: 1$ indicates a direct formation of $\mathrm{NiS}$. The front moves very slowly into the particle; even after $60 \mathrm{~h}$, only an $0.8 \mathrm{~mm}$ thick NiS layer from the particle edge has been formed.

These observations can be explained if one assumes that formation and growth of the reaction front during sulfidation at high temperatures depends on the diffusion through the already formed nickel sulfide layer. Such a behavior can be described by a shrinking core model (Jess and Wasserscheid, 2013). Here, the loading level, $X_{\mathrm{B}}$, can be calculated by Eq. (3):

$$
\frac{t}{C_{1}}=X_{\mathrm{B}}+\left(1-X_{\mathrm{B}}\right) \ln \left(1-X_{\mathrm{B}}\right) .
$$


a)

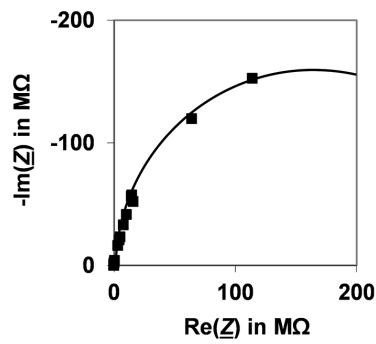

b)

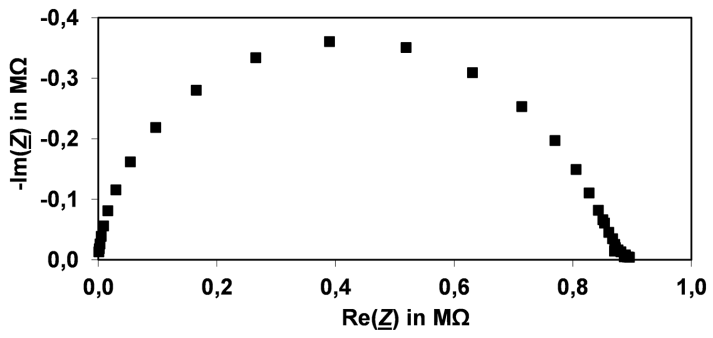

Figure 3. Typical Nyquist representation of the impedance of the single-particle sensor between $1 \mathrm{~Hz}$ and $1 \mathrm{MHz} . T=400^{\circ} \mathrm{C}$. (a) Catalyst particle in the reduced state $\left(10 \% \mathrm{H}_{2}\right.$ in $\mathrm{N}_{2}$ ). (b) Catalyst particle is sulfidized for 1 min in $500 \mathrm{ppm}_{2} \mathrm{~S}$ in $\mathrm{N}_{2}$.

a)

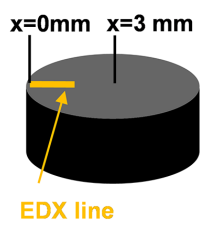

b)

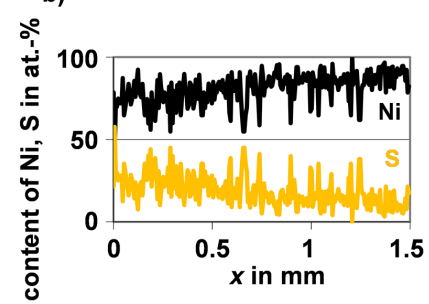

c)

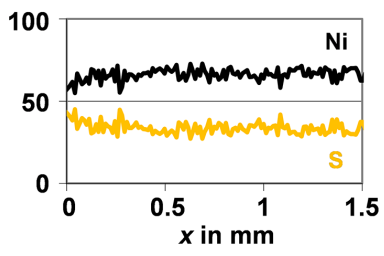

Figure 4. EDX: (a) sketch of the EDX line scan direction; (b) sample sulfidized for $6 \mathrm{~h}$ at $100{ }^{\circ} \mathrm{C}$ and $1000 \mathrm{ppm} \mathrm{H}_{2} \mathrm{~S}$; (c) same conditions after sulfidation of $60 \mathrm{~h}$.

In Eq. (3), $t$ means the sulfidation time and the factor $C_{1}$ can be calculated from Eq. (4):

$C_{1}=\frac{\rho_{\mathrm{B}} \cdot r_{\mathrm{P}}^{2}}{4 \cdot M_{\mathrm{B}} \cdot D_{\mathrm{eff}} \cdot c_{\mathrm{g}}}$.

In Eq. (4), $\rho_{\mathrm{B}}$ is the nickel fraction of the catalyst volume, $r_{\mathrm{P}}$ is the radius of the catalyst particle, $M_{\mathrm{B}}$ is the molar mass of nickel, $D_{\text {eff }}$ is the effective diffusion coefficient of $\mathrm{H}_{2} \mathrm{~S}$, and $c_{\mathrm{g}}$ denotes the gas concentration, here of $\mathrm{H}_{2} \mathrm{~S}$.

The EDX data from a sulfidation at $300{ }^{\circ} \mathrm{C}$ with $500 \mathrm{ppm}$ $\mathrm{H}_{2} \mathrm{~S}$ were taken for different sulfidation periods, to verify whether the sulfur incorporation into the catalyst particle can be quantitatively described by this model. A full loading level in Fig. 6 means that all nickel is converted into NiS.

For the model, $\rho_{\mathrm{B}} \approx 606 \mathrm{~kg} \mathrm{~m}^{-3}, \quad r_{\mathrm{P}} \approx 0.003 \mathrm{~m}$, $M_{B} \approx 0.059 \mathrm{~kg} \mathrm{~mol}^{-1}$, and $c_{\mathrm{g}} \approx 0.0105 \mathrm{~mol} \mathrm{~m}^{-3}$ were used. In order to calculate $D_{\text {eff }}$, the equation from Froment and Bischoff (1990) was applied. It is valid if a high micro and macro porosity exists in the catalyst (Kernchen et al., 2007).

$D_{\text {eff }}=\varepsilon_{M}^{2} D_{M}+\frac{\varepsilon_{\mu}^{2}\left(1+3 \cdot \varepsilon_{M}\right)}{1-\varepsilon_{M}} \cdot D_{\mu}$

The porosity $(\varepsilon)$ of the catalyst was $54 \%$ (Müller, 2011). To simplify the calculation, the porosity is split into equal parts for micro porosity $\left(\varepsilon_{\mu}\right)$ and macro porosity $\left(\varepsilon_{M}\right)$ with the respective diffusion coefficients, $D_{\mu}$ (Knudsen diffusion; average pore size diameter $8 \mathrm{~nm}$, measured by BET) and
$D_{\mathrm{M}}$ (gas diffusion). With these values $D_{\text {eff }}$ is estimated to be $1.2 \cdot 10^{-6} \mathrm{~m}^{2} \mathrm{~s}^{-1}$. For a sulfidation with $500 \mathrm{ppm}_{2} \mathrm{~S}$ at $300^{\circ} \mathrm{C}$, EDX data and the shrinking core model curve coincide well (Fig. 6).

\subsection{Impedance measurement results}

Knowing the sulfidation kinetics, the conductivity of singleparticle sensors was examined. In Fig. 7, the typical behavior of the conductivity of a single catalyst particle during sulfidation is shown, here exemplarily for $400{ }^{\circ} \mathrm{C}$ and $500 \mathrm{ppm}_{2} \mathrm{~S}$. At the beginning, in the reduced state, a particle has a low conductivity, $\sigma$. When applying high $\mathrm{H}_{2} \mathrm{~S}$ concentrations up to $1000 \mathrm{ppm}$ especially at a high temperature of up to $400^{\circ} \mathrm{C}$, the conductivity increases very quickly. During this process, nickel sulfides are formed on the catalyst surface according to XRD (X-ray diffraction) and EDX data. From the literature it is well-known that nickel sulfides exhibit a metallic conductivity almost as high as nickel (Dharmaprakash, 1996). Therefore, at first glance, during sulfidation one would have expected a small decrease in conductivity but not a conductivity increase by decades. A similar phenomenon for a $\mathrm{Ni}$ catalyst when exposed to $\mathrm{H}_{2} \mathrm{~S}$ has already been described by Bjorklund and Lundström (1983), who studied the electrical response of several supported $\mathrm{Ni}$ and Co catalysts during reaction.

In order to investigate this behavior further and to set up an initial theory, the sulfidation temperature was varied, always keeping the $\mathrm{H}_{2} \mathrm{~S}$ concentration at $1000 \mathrm{ppm}$. The results are 


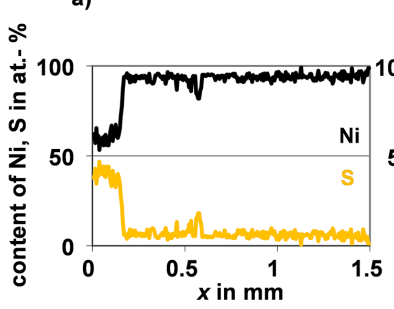

b)

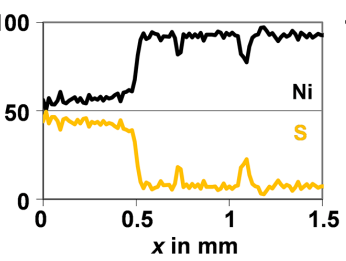

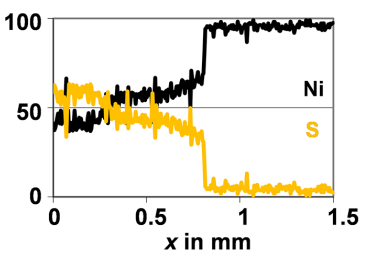

Figure 5. EDX results for samples sulfidized at $300^{\circ} \mathrm{C}$ in $500 \mathrm{ppm} \mathrm{H}_{2} \mathrm{~S}$ in $\mathrm{N}_{2}$; (a) sulfidation time $2 \mathrm{~h}$; (b) $24 \mathrm{~h}$; (c) $60 \mathrm{~h}$.

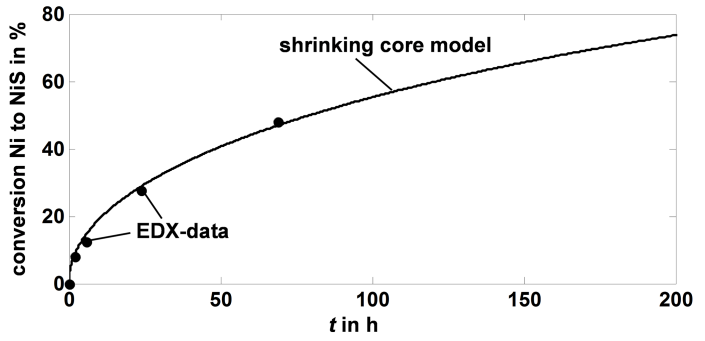

Figure 6. Amount of NiS plotted against the sulfidation time $\left(T=300{ }^{\circ} \mathrm{C} ; c_{\mathrm{H}_{2} \mathrm{~S}}=500 \mathrm{ppm}\right)$. The line is calculated with the shrinking core model (Eq. 5). Dots are determined by EDX.

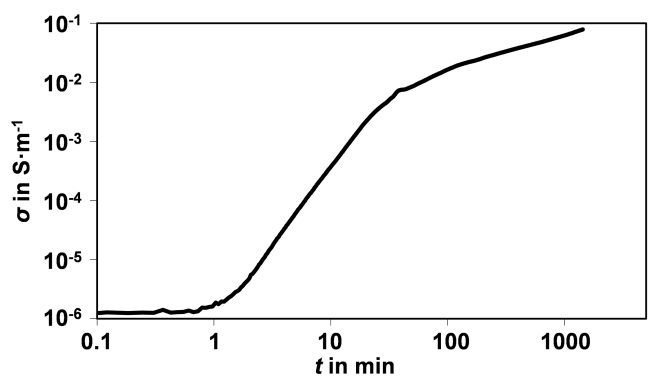

Figure 7. Example for the conductivity response of a single catalyst pellet during sulfidation (data: $500 \mathrm{ppm}_{2} \mathrm{~S}$ in $\mathrm{N}_{2}, 400^{\circ} \mathrm{C}$ ).

shown in Fig. 8. To better compare the results of different temperatures, a relative conductivity, $\sigma_{\text {rel }}$, was calculated by Eq. (6):

$\sigma_{\text {rel }}=\frac{\sigma}{\sigma_{0}}$,

where $\sigma_{0}$ is the conductivity of each sensor particle after preconditioning, right before sulfidation. It is in the range of about $10^{-6} \mathrm{~S} \cdot \mathrm{m}^{-1}$. The conductivity increases almost directly with the beginning of sulfur poisoning at $300^{\circ} \mathrm{C}$ and above. During further sulfidation, the slopes of the curves decrease only slightly and an overall slope of almost 2 can be found in the double-logarithmic representation. In contrast, at lower temperatures, the curve shape changes to an S-like behavior. The lower the temperature, the longer the conductivity remains constant before it starts to increase. The increase in conductivity, i.e., the decreasing electrical resis-

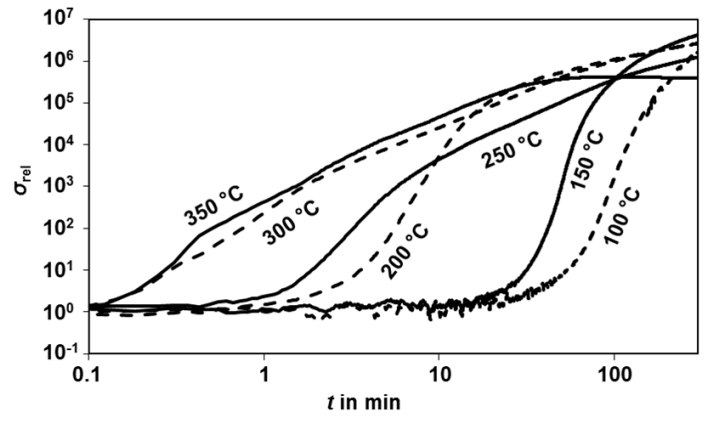

Figure 8. Relative conductivity during sulfidation under $1000 \mathrm{ppm}$ $\mathrm{H}_{2} \mathrm{~S}$ in $\mathrm{N}_{2}$ at different temperatures. For the temperature curves at 100 and $150^{\circ} \mathrm{C}$, the measured values were averaged over 10 points.

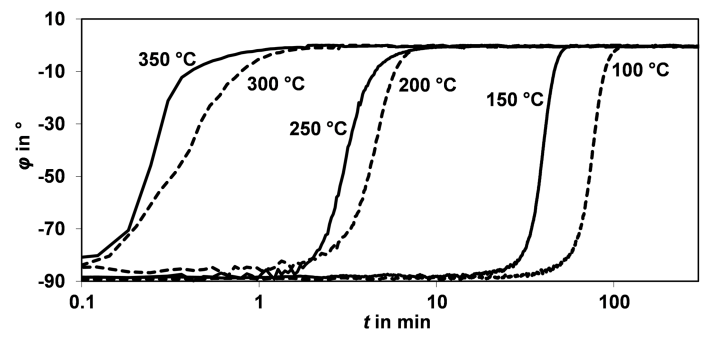

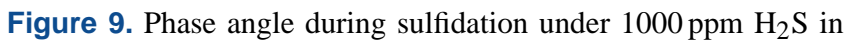
$\mathrm{N}_{2}$ at different temperatures. For the temperature curves at 100 and $150^{\circ} \mathrm{C}$, the measured values were averaged over 10 points.

tance, goes along with a transition from capacitive to ohmic behavior. This is reflected by the changing phases $\phi$ from almost -90 to almost $0^{\circ}$ (see Fig. 9).

To illustrate the effect of temperature, a characteristic time, $t_{\mathrm{c}}$, is introduced. It denotes the sulfidation start. From Fig. $8, t_{\mathrm{c}}$ can be derived by the intersection of the baseline with the initial slope of the sulfidation. From the behavior of the phase angle (Fig. 9), the characteristic times are defined as the points in time, when the phase angles reached $-45^{\circ}$. These two characteristic times are plotted for different temperatures against the reciprocal sulfidation temperature in Fig. 10 (Arrhenius-type representation).

As mentioned above, one would not have expected a conductivity increase during sulfidation, since $\mathrm{Ni}$ is a better electrical conductor than $\mathrm{NiS}$ or $\mathrm{Ni}_{3} \mathrm{~S}_{2}$, although both sulfides are 


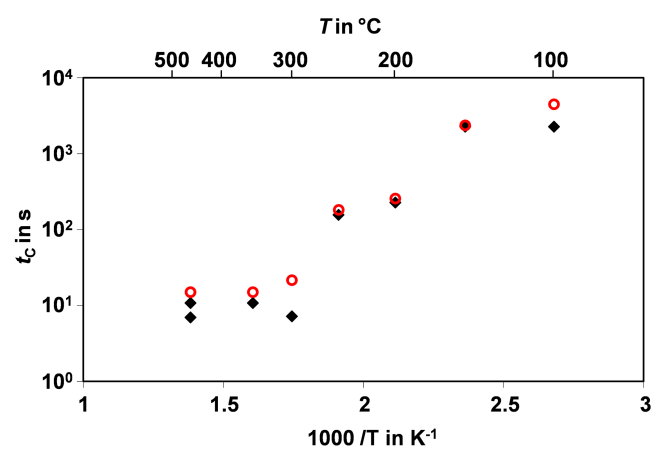

Figure 10. Characteristic time $\left(t_{\mathrm{c}}\right)$ at different temperatures. The black squares symbolize the characteristic time from the conductivity curves at the beginning of sulfur poisoning. The open circles denote the characteristic time from derived from the phase curves $\left(\phi=-45^{\circ}\right)$.

metal-like conductors. Hence, one needs an explanation for the conductivity increase that occurs by decades during sulfidation.

To explain the low conductivity in the reduced state, we assume that $\mathrm{Ni}$ is so highly dispersed on the porous catalyst particle that no conductive paths exist between the electrodes. We further assume that during sulfidation, the volume expansion from nickel to nickel sulfide forms conductive percolation paths (molar volume of nickel $\approx 6.6 \mathrm{~cm}^{3} \cdot \mathrm{mol}^{-1}$ and nickel sulfide $\approx 16.5 \mathrm{~cm}^{3} \cdot \mathrm{mol}^{-1}$; Blachnik, 1998). Similar effects are reported in literature for $\mathrm{CuO}$ nanofibers when exposed to $\mathrm{H}_{2} \mathrm{~S}$ (Hennemann et al., 2012). Here, $\mathrm{CuO}$ reacts to copper sulfide. After a certain time, a fast increase of the conductivity occurs. The authors attribute this to the formation of percolation paths between the single nanofibers. A similar effect, namely a strong conductivity increase, is observed for $\mathrm{Pd}$ exposed with $\mathrm{H}_{2}$. This effect is also attributed to a volume expansion that leads to percolation effects (Dankert and Pundt, 2002). The study of Bjorklund et al. (1985) showed that metal crystallites of supported catalysts increase in $\mathrm{O}_{2}$ and become smaller in $\mathrm{H}_{2}$. Due to this volume change, conductive paths through the catalyst form.

The percolation is a statistical probability for building clusters in a system. At a specific limit of one substance, a cluster is building between the boundaries of the system. This limit is called the percolation threshold. During sulfidation, it can be noticed through the large conductivity increase of the sensor, which can be explained very well with the percolation theory (Cruz-Estrada and Folkes, 2002). In Fig. 7, the conductivity increases very rapidly at high temperatures. During this time, only a small nickel sulfide layer is built up; compare with Fig. 6. This small layer suffices to build enough conductive paths that the percolation threshold is reached. Also in Fig. 10 a quick sulfidation is seen by the small characteristic times at temperatures above $300^{\circ} \mathrm{C}$. If the temperature is now decreased, the loading mechanism changes. At $100{ }^{\circ} \mathrm{C}$, sulfidation is slower and almost homogeneous over the catalyst particle (Fig. 4). This suggests that the first conductive paths are built slower. Therefore, the characteristic time in Fig. 10 increases at lower temperatures. Based on the different loading mechanism at different temperatures it is also possible that the same sulfur amount does not result in the same conductivity value due to different numbers of conductive paths. This could be a reason for the different curve progression in Fig. 8.

\section{Conclusion and outlook}

The applied single-particle sensor can be used to measure directly the sulfur loading of a catalyst (e.g., of a fixed bed). This method is therefore an ideal supplement and extension of the determination of the coking degree of catalyst pellets by a similar setup (Müller et al., 2010a, c). There, coking and resistance change occur in the same timely dimensions but instead of sulfidation coke is deposited on the (internal) surface of the catalyst. This can be measured in almost the same manner with the sulfur sensor at low temperatures $\left(<150^{\circ} \mathrm{C}\right)$. In contrast to that, we very precisely see the onset of the sulfidation by a pronounced conductivity increase and a phase angle change from capacitive insulating to ohmic behavior at high temperatures $\left(>200^{\circ} \mathrm{C}\right)$. This is explained by a direct formation of conductive percolation paths in the nickel sulfide layer of the catalyst particles. They have their origin in the volume increase of nickel when reacting with sulfur to nickel sulfides. The sensor clearly indicates how the sulfidation process increases with temperature.

Further work will be dedicated to setup a mathematical model that describes quantitatively the correlation between conductivity and sulfidation degree. In additional experiments, samples with much lower Ni contents will be investigated. Since the volume fraction of formed NiS will then be lower, we expect not to find a strong conductivity increase, because no percolation paths should be formed. This should support the percolation theory.

In a second research direction, it shall be investigated whether the promising results with a microwave cavity perturbation method, which allows detecting the degree of coke on catalyst pellets contactless (Müller et al., 2011), can be transferred to the sulfidation process.

Acknowledgements. The authors A. Jess and R. Moos are indebted to the German Research Foundation (DFG) for financial support (Je 257/12-2, Mo 1060/5-2, respectively).

Edited by: A. L. Spetz

Reviewed by: three anonymous referees 


\section{References}

Bartholomew, C. H., Weatherbee, G. D., and Jarvi, G. A.: Sulfur poisoning of nickel methanation catalysts, J. Catal., 60, 257-269, 1979.

Blachnik, R.: Taschenbuch für Chemiker und Physiker: Elemente, anorganische Verbindungen und Materialien, Minerale, Band 3, Springer, Heidelberg, 644 pp., 1998.

Bjorklund, R. B. and Lundström, I.: Electrical Conductance of Catalysts in Contact with Gaseous Reactants, J. Catal., 79, 314-326, 1983.

Bjorklund, R. B., Söderberg, D., and Lundström, I.: Electricalconductance Responses of Catalysts Exposed to Pulses of $\mathrm{H}_{2}$ and $\mathrm{O}_{2}$, Journal of Chemical Society, Faraday Transactions, 81, 1715-1724, 1985.

Cruz-Estrada, R. H. and Folkes, M. J.: Structure formation and modelling of the electrical conductivity in SBS-polyaniline blends: Part I: Percolation theory approach, J. Mater. Sci. Lett., 21, 14271429, 2002.

Dankert, O. and Pundt, A.: Hydrogen-induced percolation in discontinuous films, Appl. Phys. Lett., 81, 1618-1620, 2002.

Dharmaprakash, S. M.: Synthesis and electrical conductivity of nickel sulphide, Cryst. Res. Technol., 31, 49-53, 1996.

Froment, G. F. and Bischoff, K. B.: Chemical reactor analysis and design, John Wiley \& Sons, New York, 148 pp., 1990.

Hennemann, J., Sauerwald, T., Kohl, C.-D., Wagner, T., Bognitzki, M., and Greiner, A.: Electrospun copper oxide nanofibers for $\mathrm{H}_{2} \mathrm{~S}$ dosimetry, Phys. Status Solidi A, 209, 911-916, 2012.

Jess, A. and Depner, H.: Kinetics of nickel-catalyzed purification of tarry fuel gases from gasification and pyrolysis of solid fuels, Fuel, 78, 1369-1377, 1999.
Jess, A. and Wasserscheid, P.: Chemical Technology: An Integrated Textbook, Wiley-VCH, Weinheim, 280 pp., 2013.

Kernchen, U., Etzold, B., Korth, W., and Jess, A.: Solid catalyst with ionic liquid layer (SCILL) - A new concept to improve selectivity illustrated by hydrogenation of cyclooctadiene, Chem. Eng. Technol., 30, 985-994, 2007.

Macdonald, J.: Impedance Spectroscopy, John Wiley \& Sons, New York, p. 15, 1987.

Müller, N.: Direkte Bestimmung von Koksdepositen auf Festbettkatalysatoren durch elektrische Sensoren, Bayreuther Beiträge zur Sensorik und Messtechnik, Shaker Verlag, Aachen, 143 pp. 2011.

Müller, N., Kern, C., Moos, R., and Jess, A.: Direct detection of coking and regeneration of single particles and fixed bed reactors by electrical sensors, Appl. Catal. A-Gen., 382, 254-262, 2010a.

Müller, N., Jess, A., and Moos, R.: Direct detection of coke deposits on fixed bed catalysts by electrical sensors, Sensors Actuat. BChem., 144, 437-442, 2010 b.

Müller, N., Moos, R., and Jess, A.: In-situ monitoring of coke deposits during coking and regeneration of solid catalysts by electrical impedance-based sensors, Chem. Eng. Technol., 33, 103112, 2010c.

Müller, N., Reiß, S., Fremerey, P., Jess, A., and Moos, R.: Initial tests to detect quantitatively the coke loading of reforming catalysts by a contactless microwave method, Chem. Eng. Process., 50, 729-731, 2011.

Oudar, J.: Sulfur Adsorption and Poisoning of Metallic Catalysts, Cataly. Rev., 22, 171-195, 1980.

Schönauer, D. and Moos, R.: Detection of water droplets on exhaust gas sensors, Sensors Actuat. B-Chem., 148, 624-629, 2010. 